\title{
Controllable Group Delay in a $\theta$-Shaped Microfiber Resonator with Coupled-Resonator-Induced Transparency
}

\author{
Yiyang Luo ${ }^{1 \dagger}$, Zhilin $\mathrm{Xu}^{1,3 \dagger}$, Qizhen Sun ${ }^{1,2+*}$, Borui $\mathrm{Li}^{1}$, Perry Ping Shum ${ }^{3}$, Lin Zhang ${ }^{2}$ and Deming Liu ${ }^{1}$ \\ ${ }^{1}$ School of Optical and Electronic Information, and National Engineering Laboratory for Next Generation Internet Access System, Huazhong \\ University of Science and Technology, Wuhan 430074, Hubei, P. R. China; \\ ${ }^{2}$ Aston Institute of Photonic Technologies, Aston University, B4 7ET, United Kingdom; \\ ${ }^{3}$ COFT, School of Electrical and Electronic Engineering, Nanyang Technological University, Singapore. \\ \% The authors are equally contributed to this paper \\ *E-mail address: qzsun@mail.hust.edu.cn; q.sun2@aston.ac.uk
}

\begin{abstract}
The control of Light velocity is theoretically and experimentally demonstrated in a $\theta$ shaped microfiber resonator with coupled-resonator-induced transparency. By adjusting the structure parameters, group delays from $-60 \mathrm{ps}$ to $200 \mathrm{ps}$ are achieved in the all-fiber resonator. OCIS codes: (060.2310) Fiber optics; (230.5750) Resonators; (060.2280) Fiber design and fabrication.
\end{abstract}

\section{Introduction}

Controlling the light velocity has attracted tremendous attentions due to both the fundamental research interest and the numerous applications such as optical information storage, optical buffers, tunable optical delay lines, and data synchronization [1-6]. Especially, technique of electromagnetically induced transparency (EIT) is proved to be an effective way to freeze light [1]. However, serious EIT phenomena coming from coherent interactions of light with multilevel atoms usually require extreme conditions, which hinder their practical applications [1]. As a substitution, various techniques analogous to EIT have also achieved significant progress in the light-velocity control, particularly including stimulated Brillouin scattering [2], stimulated Raman scattering [3], photonics crystal-based resonance structure [4], and coupled-resonator-induced transparency (CRIT) [5, 6].

In the past several years, micro/nano fibers (MNFs) drawn from standard single mode fibers (SMFs) have gained considerable advancements for their distinct advantages of large evanescent field, good mechanical property, easy coupling to other fiber components, and significantly lower loss than lithographically fabricated waveguides. Moreover, various microfiber resonators with different structures have been experimentally demonstrated for versatile applications, also paving another way to control the velocity of light based on the all-optical analog of EIT. Particularly, previous literatures $[7,8]$ have obtained 25ps fast light in a microfiber knot resonator and 70ps slow light in a microfiber double-knot resonator respectively. However, tunable group delays from slow light to fast light have never demonstrated in a microfiber resonator, to the best of our knowledge.

In this paper, we propose a compact and reconfigurable $\theta$-shaped microfiber resonator for all-fiber lightvelocity control based on CRIT. Benefiting from the CRIT induced by this specific resonant structure, both slow light and fast light can be achieved by choosing appropriate structure parameters. Theoretical analysis is carried out to investigate the influence of the resonator parameters on the group delay. Experimental results verify that the tunable group delays from 60 ps fast light to 200 ps slow light can be achieved via simply adjusting the coupling regions and the size of the $\theta$-shaped microfiber resonators.

\section{Physical design and theoretical analysis}

The schematic diagram of the $\theta$-shaped microfiber resonator is shown in Fig. 1, where an elliptical cavity is divided by a bridge microfiber into the shape of character " $\theta$ ". Due to the linking function of the bridge microfiber, clockwise $(\mathrm{CW})$ mode and counterclockwise $(\mathrm{CCW})$ mode conjunctly oscillate in the cavity and destructively interfere with each other, consequently inducing the CRIT, which is analogous to the EIT. The reflected part and the transmitted part of the interfered light are output from port 1 and port 8 , respectively. Since the transmitted and reflected light experience the same propagation path, the effective phase shifts and the group delays are actually the same. Owing to the ultra-compactness, the reflected output of the proposed $\theta$-shaped microfiber resonator is adopted to investigate the group delay. For simplification, we assume that the two coupling areas (I, II) possess the identical coupling efficiency $k$ and excess loss $\gamma$. Then the reflected transfer function can be obtained by equation (1). Consequently, the effective phase shift and the group delay can be derived by equation (2), where $\beta$ and $n_{\text {eff }}$ denote the propagation constant and the effective index in the $\theta$-shaped microfiber resonator; $l_{0}, l_{1}$ and $l_{2}$ respectively represent the distances from port 3 to port6, from port 2 to port 5 , and from port 7 to port 4 , as well as $L=l_{1}+l_{2}$; 
and $c$ is the light velocity in vacuum, respectively. In the following analysis, the off-resonance is taken as the zero point of the group delay.

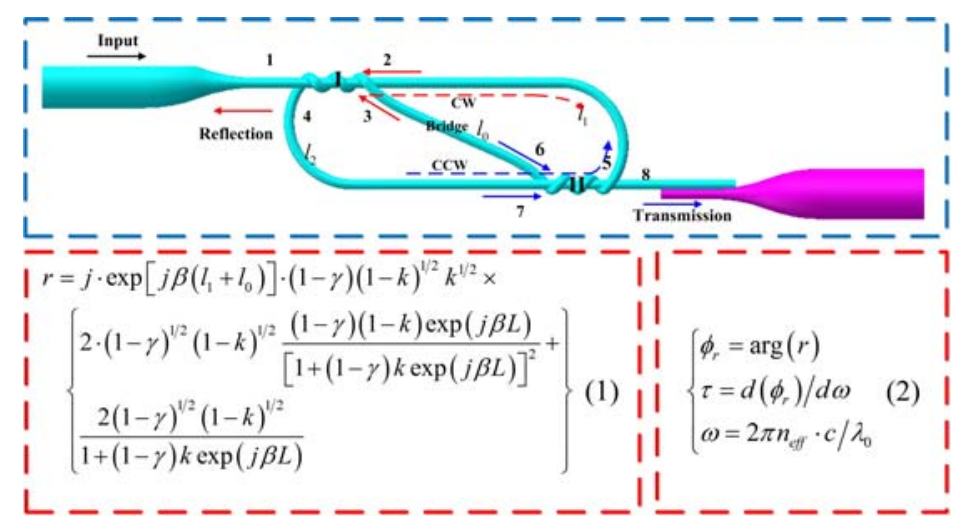

Fig. 1. Schematic diagram of the proposed $\theta$-shaped microfiber resonator and related equations.

The effects of coupling efficiency on reflected spectrum and group delay are respectively illustrated in Figs. 2(a) and 2(b), where microfiber diameter, cavity length $L$ and excess loss $\gamma$ are fixed at $1 \mu \mathrm{m}, 4 \mathrm{~mm}$ and 0.2 , respectively. Fig. 2(b) shows that fast light or slow light occurs due to the strong normal or anomalous dispersions near the resonant wavelength. With an increasing coupling efficiency from 0.1 to 0.9 , the group delay gradually turns from fast light to slow light. Similarly, the influence of excess loss is also presented in Figs. 2(c) and 2(d). When the excess loss is increased from 0.05 to 0.5 with a fixed coupling efficiency of 0.3 , only fast-light behaviors are obtained accompanying with a significantly increased group delay as well as a decreased extinction ratio of the reflected spectrum. Moreover, Figs 2(e) and 2(f) reveal that cavity length of the resonator plays a crucial role in the group delay as well. It can be seen that the free space range (FSR) and the group delay are respectively inversely and directly proportional to the cavity length of the resonator. However, the long cavity length will definitely augment the whole size of the resonator. Therefore, there is a trade-off between the group delay and the size of $\theta$-shaped microfiber resonator in practical applications.
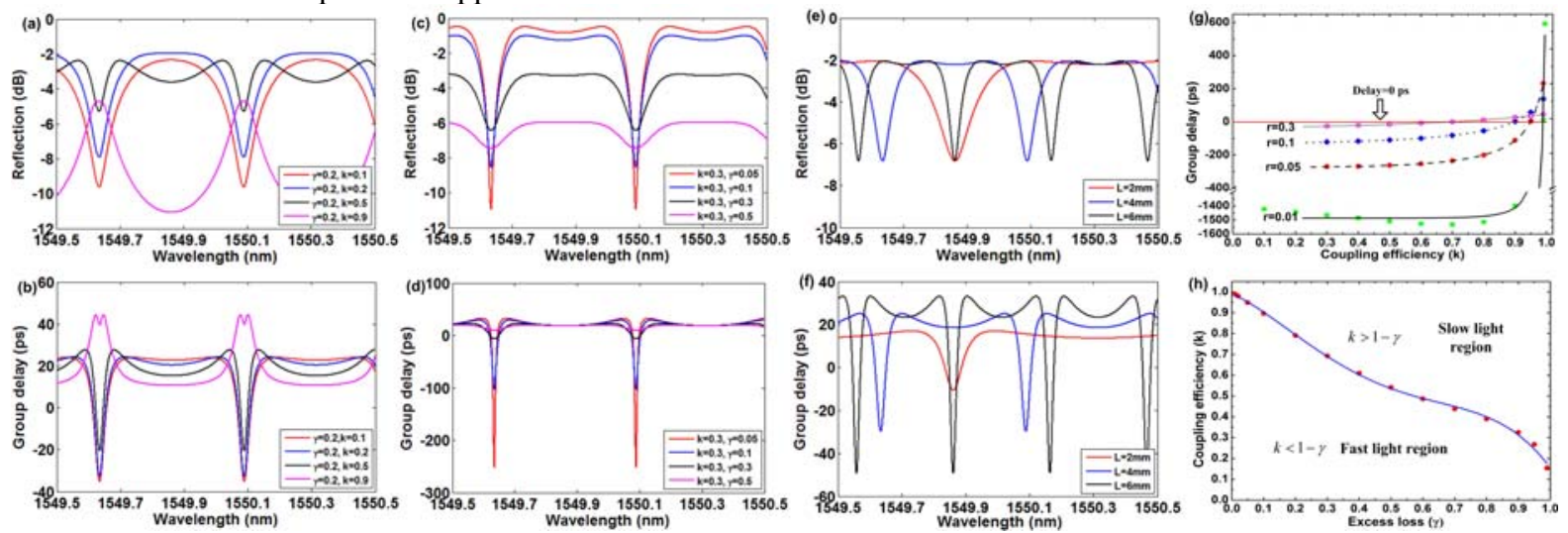

Fig. 2. The reflection spectra and group delays as functions of wavelength evolve as (a) (b) coupling efficiency, (c) (d) excess loss and (e) (f) cavity length. (g) Group delay as a function of $k$ under different $\gamma$. (h) Critical coupling efficiency and excess loss for slow light and fast light.

Furthermore, a profounder insight into the effects of coupling efficiency and excess loss on the group delay is also revealed. Fig. 2(g) illustrates the group delay as a function of coupling efficiencies increased from 0.1 to 0.9 with different excess losses of $0.01,0.05,0.1$ and 0.3 , where the microfiber diameter and cavity length are fixed at $1 \mu \mathrm{m}$ and $4 \mathrm{~mm}$ respectively. Apparently, the group delay of $-1400 \mathrm{ps}$ (fast light) and 600ps (slow light) would be generated when the excess loss is decreased to 0.01.It can be inferred that the transition points should exist where the group delay turns from fast light to slow light. Specifically, we extract the transient points and display them in the coupling efficiency-excess loss plane, as shown in Fig. 2(h). By fitting the transient points with polynomial curve, the whole coupling efficiency-excess loss plane is divided into two parts, i.e. the fast light region and the slow light region. Therefore, by tuning the coupling efficiency and the excess loss, different group delays can be obtained.

\section{Experiments and results}


The experimental setup for group-delay measurement is depicted in Fig. 3(a). A $\theta$-shaped microfiber resonator with the size of $2 \mathrm{~mm} \times 1 \mathrm{~mm}$ is fabricated from a microfiber with diameter of $2 \mu \mathrm{m}$, and the reflected output is employed to verify the group-delay properties. Particularly, a tunable laser with a linewidth of $100 \mathrm{kHz}$ is modulated into $1.7 \mathrm{GHz}$ sinusoidal pulse by an electro-optic modulator (EOM). After being amplified by an erbium-doped fiber amplifier (EDFA), the laser pulse subsequently passes through the microfiber resonator and a variable optical attenuation (VOA). Additionally, an optical spectrum analyzer (OSA) is used to monitor the reflection spectra, and a digital storage oscilloscope (DSO) is utilized to record the pulses, as well as to drive the EOM. Firstly, the wavelength of the laser pulse is successively tuned from the off-resonant wavelength (labeled A) to the on-resonant wavelength (labeled B) as presented in Fig. 3(b). Through point-by-point scanning of the reflection spectrum, group delay as a function of wavelengths is also illustrated in Fig. 3(b), where zero point is set at the off-resonant wavelength. Assisted with Lorentz fitting, the maximum group delay of $85 \mathrm{ps}$ emerges at the on-resonant wavelength of $1560.01 \mathrm{~nm}$ while decreases on both sides. Fig. 3(c) reveals that pulse delay, namely slow light, can be distinctly observed at the on-resonant wavelength without significant pulse distortion.

Meanwhile, the group-delay properties of the $\theta$-shaped microfiber resonator with different coupling efficiencies, excess losses, and cavity lengths are experimentally studied as well, which are realized by micro-mechanical adjustment of the resonator structure. Figs. 3(d) and 3(e) present various reflection spectra of the microfiber resonator, as well as the corresponding pulse temporal profiles at the off-resonant and on-resonant wavelengths. It is evident that both fast light and slow light can be achieved via simply adjusting the coupling regions and the size of the $\theta$-shaped microfiber resonators. The obtained group delay are respectively $-60 \mathrm{ps},-50 \mathrm{ps},-40 \mathrm{ps}, 130 \mathrm{ps}$ and $200 \mathrm{ps}$, straightforwardly demonstrating the group-delay tunability of the $\theta$-shaped microfiber resonator.
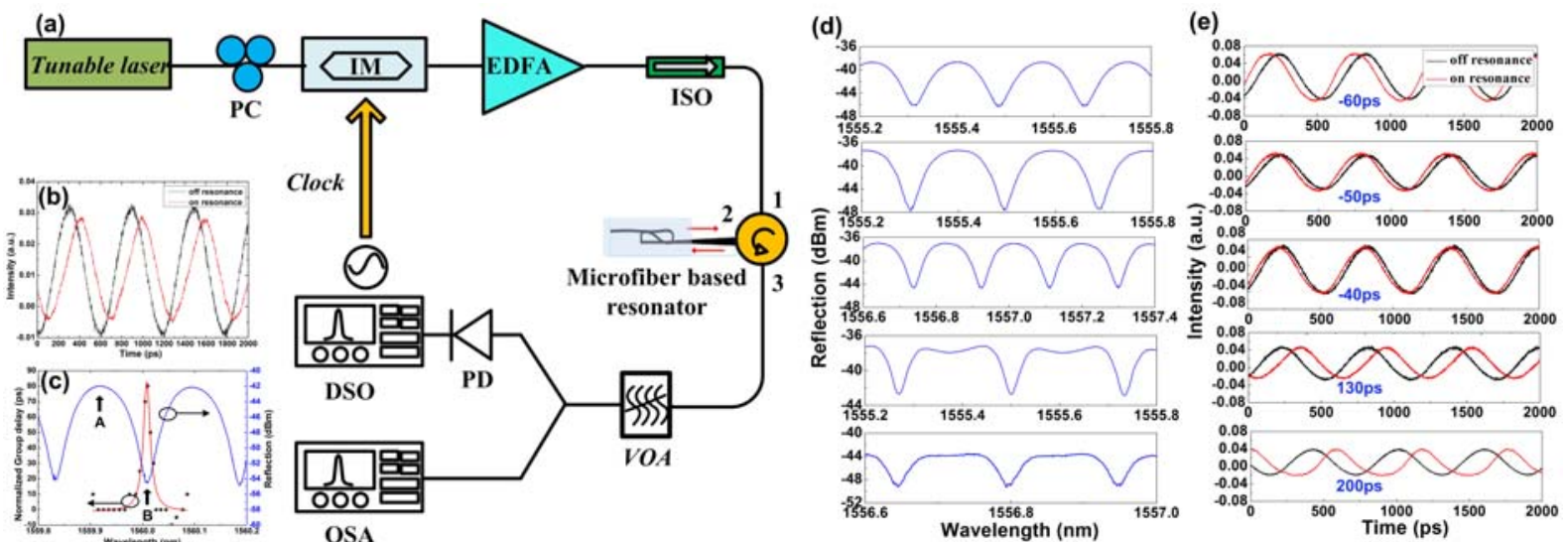

Fig. 3. (a) Schematic of the experimental setup for group-delay measurement. (b) Measured group delay as a function of wavelength (red curve), as well as corresponding reflection spectrum (blue curve). (c) Oscilloscope traces of the pulses showing slow light. (d) Reflection spectra of the $\theta$-shaped microfiber resonator with different structure parameters. (e) Corresponding pulse temporary profiles at the off-resonant wavelength and on-resonant wavelength.

\section{Conclusions}

A $\theta$-shaped microfiber resonator with CRIT is proposed and demonstrated for light velocity control. Theoretical analysis reveals that the group delay could be extensively tuned from -1400ps (fast light) to 600ps (slow light) by adjusting the coupling efficiencies, excess losses and cavity lengths of the resonator. Both of the fast light up to 60ps and the slow light up to 200ps are achieved in experiment. The $\theta$-shaped microfiber resonator can be served as a compact, tunable and all-fiber light-velocity controller for optical storage, optical buffers, data synchronization, etc.

Acknowledgment: This work is supported by the National Natural Science Foundation of China (No. 61290315, No. 61275004), and the European Commission's Marie Curie International Incoming fellowship (No. 328263) .

\section{References}

[1] L. V. Hau, S. E. Harris, Z. Dutton, and C. H. Behroozi, Nature 397, 594 (1999).

[2] Kim, J., Kuzyk, M. C., Han, K., Wang, H., and G. Bahl, Nature Phys. 11, 275-280 (2015).

[3] M. S. Blair and K. Zheng, Opt. Express 14, 1064-1069 (2006).

[4] X. Yang, M. Yu, D. L. Kwong, and C. H. Wong, Phys. Rev. Lett. 102, 173902 (2009).

[5] K. Totsuka, N. Kobayashi, and M. Tomita, Phys. Rev. Lett. 98, 213904, (2007).

[6] Q. Xu, J. Shakya, and M. Lipson, Opt. Express, 14, 6463-6468 (2006).

[7] T. Wang, X. Li, F. Liu, W. Long, Z. Zhang, L. Tong, and Y. Su, Opt. Express, 18, 16156-16161 (2010).

[8] Y. Xu, L. Ren, J. Liang, C. Ma, Y. Wang, X. Kong, X. Lin, J. Appl. Phys. 118, 073105 (2015). 